\title{
Different Faces of Generalized Holographic Dark Energy
}

\author{
${\text { Shin'ichi Nojiri }{ }^{1,2} \text { (D), Sergei D. Odintsov }}^{3,4}$ and Tanmoy Paul ${ }^{5,6, *(D)}$ \\ 1 Department of Physics, Nagoya University, Nagoya 464-8602, Japan; nojiri@gravity.phys.nagoya-u.ac.jp \\ 2 Kobayashi-Maskawa Institute for the Origin of Particles and the Universe, Nagoya University, \\ Nagoya 464-8602, Japan \\ 3 ICREA, Passeig Luis Companys, 23, 08010 Barcelona, Spain; odintsov@ieec.uab.es \\ 4 Institute of Space Sciences (IEEC-CSIC) C. Can Magrans s/n, 08193 Barcelona, Spain \\ 5 Department of Physics, Chandernagore College, Hooghly 712 136, India \\ 6 International Laboratory for Theoretical Cosmology, TUSUR, 634050 Tomsk, Russia \\ * Correspondence: pul.tnmy9@gmail.com
}

check for

updates

Citation: Nojiri, S.; Odintsov, S.D.; Paul, T. Different Faces of Generalized Holographic Dark Energy. Symmetry 2021, 13, 928. https://doi.org/10.3390/sym13060928

Academic Editor: Tomohiro Inagaki

Received: 20 April 2021

Accepted: 18 May 2021

Published: 23 May 2021

Publisher's Note: MDPI stays neutral with regard to jurisdictional claims in published maps and institutional affiliations.

Copyright: (C) 2021 by the authors Licensee MDPI, Basel, Switzerland. This article is an open access article distributed under the terms and conditions of the Creative Commons Attribution (CC BY) license (https:/ / creativecommons.org/licenses/by/ $4.0 /)$.

\begin{abstract}
In the formalism of generalized holographic dark energy (HDE), the holographic cut-off is generalized to depend upon $L_{\mathrm{IR}}=L_{\mathrm{IR}}\left(L_{\mathrm{p}}, \dot{L}_{\mathrm{p}}, \ddot{L}_{\mathrm{p}}, \cdots, L_{\mathrm{f}}, \dot{L}_{\mathrm{f}}, \cdots, a\right)$ with $L_{\mathrm{p}}$ and $L_{\mathrm{f}}$ being the particle horizon and the future horizon, respectively (moreover, $a$ is the scale factor of the Universe). Based on such formalism, in the present paper, we show that a wide class of dark energy (DE) models can be regarded as different candidates for the generalized HDE family, with respective cut-offs. This can be thought as a symmetry between the generalized HDE and different DE models. In this regard, we considered several entropic dark energy models—such as the Tsallis entropic DE, the Rényi entropic DE, and the Sharma-Mittal entropic DE-and found that they are indeed equivalent with the generalized HDE. Such equivalence between the entropic DE and the generalized HDE is extended to the scenario where the respective exponents of the entropy functions are allowed to vary with the expansion of the Universe. Besides the entropic DE models, the correspondence with the generalized HDE was also established for the quintessence and for the Ricci DE model. In all the above cases, the effective equation of state (EoS) parameter corresponding to the holographic energy density was determined, by which the equivalence of various DE models with the respective generalized HDE models was further confirmed. The equivalent holographic cut-offs were determined by two ways: (1) in terms of the particle horizon and its derivatives, (2) in terms of the future horizon horizon and its derivatives.
\end{abstract}

Keywords: holographic dark energy; equivalence with generalized HDE; Tsallis dark energy; Rényi dark energy; Sharma-Mittal dark energy; quintessence dark energy; ricci dark energy

\section{Introduction}

The holographic principle originates from black hole thermodynamics and string theory and establishes a connection of the infrared cutoff of a quantum field theory, which is related to the vacuum energy, with the largest distance of this theory [1-4]. This holographic consideration is extensively applied in field cosmology, particularly when describing the dark energy (DE) era, generally as the holographic dark energy (HDE) model [5-36]. It may be stressed that instead of adding an extra term in the Lagrangian, the HDE is based on the holographic principle and on the dimensional analysis, and from this perspective, the HDE is significantly different than the other dark energy models. Apart from the dark energy era, the holographic principle has also been successfully applied to the early inflationary Universe [37-42]. Actually, during the early Universe, the size of the Universe was small, due to which, the holographic energy density was significant to triggering an inflationary scenario, and moreover, the holographic inflation was also found to be compatible with the 2018 Planck constraints. Recently, some of our authors showed that the energy density coming from the holographic principle is able to unify the early inflationary scenario with the late dark energy era in a covariant formalism [43]. From a different viewpoint, the 
application of the holographic principle was extended to the bouncing scenario in [44-48], where the holographic energy density helps to violate the energy conditions and in turn leads to a bouncing universe.

Coming back to the dark energy context, the holographic dark energy density is proportional to the inverse squared of the holographic cut-off $\left(L_{\mathrm{IR}}\right)$, which is usually assumed to be the same as the particle horizon $\left(L_{\mathrm{p}}\right)$ or the future horizon $\left(L_{\mathrm{f}}\right)$. However, the fundamental form of the $L_{\mathrm{IR}}$ is still a debatable topic in this context. It is worth mentioning that the most generalized cut-off has been proposed in [9], where in particular, the cut-off is considered to depend upon $L_{\mathrm{IR}}=L_{\mathrm{IR}}\left(L_{\mathrm{p}}, \dot{L}_{\mathrm{p}}, \ddot{L}_{\mathrm{p}}, \cdots, L_{\mathrm{f}}, \dot{L}_{\mathrm{f}}, \cdots, a\right)$, which in turn leads to the generalized version of HDE (known as "generalized HDE"). Said generalized form of $L_{\mathrm{IR}}$ immediately leads to the following question:

- $\quad$ Do there exist suitable form(s) of $L_{\mathrm{IR}}$ such that various dark energy models (including the entropic DE models) can be thought to be equivalent to the generalized HDE? If so, then what will be the equivalent form(s) of $L_{\mathrm{IR}}$ for the respective DE models?

In the present paper, we intend to address the above questions. For this purpose, we consider several entropic DE models, such as the Tsallis entropic DE [49-62], the Rényi entropic DE [63-68], and the Sharma-Mittal entropic DE [67,69,70]. Here it may be mentioned that unlike the Tsallis and Rényi entropy functions, Sharma-Mittal entropy represents a more general two-parameter function. In this regard, our investigation was carried for two cases: (1) the respective exponents of the entropy functions were treated as constant, whereas in the second case, (2) the exponents were allowed to vary with cosmic time; in particular, for the latter case, the exponents were considered to depend on the evolving Hubble parameter of the Universe. Besides the entropic DE models, the quintessence [71-75] and the Ricci DE [23,29,76,77] models were used in the analysis. In the case of the quintessence model, a non-minimally coupled scalar field with an exponential potential provides the dark energy density, whereas in the Ricci DE scenario, the space-time Ricci curvature provides the dark energy density. Both the quintessence and the Ricci DE models are in fact viable with respect to various dark energy observations. Interestingly, we show that all such entropic DE, quintessence and Ricci DE models are indeed equivalent with the generalized HDE, with suitable forms of the corresponding cut-offs.

\section{The Thermodynamics of Space-Time and Applications to Cosmology}

After the thermodynamical properties of the black hole were clarified [78,79] and it was claimed that the entropy of the black hole is proportional to the area $A$ of the horizon,

$$
S=\frac{A}{4 G}, \quad A=4 \pi r_{H}^{2},
$$

which is called Bekenstein-Hawking entropy, where $r_{H}$ is the horizon radius-and we work in units where $\hbar=k_{B}=c=1$-there were long and active studies where the connections between gravity and thermodynamics were clarified [80-82]. In the studies, we found that the FRW equations can be also regarded as the first law of thermodynamics when we consider the Bekenstein-Hawking entropy by using the cosmological apparent horizon [83-85] as a realization of the thermodynamics of space-time [80].

If, however, there are long range forces, such as the electromagnetic force and the gravitational force, we know that the systems are non-additive systems and the standard Boltzmann-Gibbs additive entropy should not be applied and we should generalize the entropy to the non-extensive Tsallis entropy [49-51], and recently there were several attempts in this regard (see [52-62]). If we apply Tsallis entropy to the black hole, instead of Bekenstein-Hawking entropy, one finds [52],

$$
S_{\mathrm{T}}=\frac{A_{0}}{4 G}\left(\frac{A}{A_{0}}\right)^{\delta} \text {. }
$$


In the above expression, $A_{0}$ is a constant and $\delta$ is the new parameter that quantifies the non-extensivity. Then if we apply Tsallis entropy by using the apparent horizon to the cosmology, the FRW equations should be modified and the modification can be regarded as the contribution from the dark energy.

In information theory, Rényi entropy is often used as the measure of the entanglement. If we apply Rényi entropy to the black hole, one finds [63-67]:

$$
S_{\mathrm{R}}=\frac{A_{0}}{G \delta} \ln \left(1+\frac{\delta}{4}\left(\frac{A}{A_{0}}\right)\right) .
$$

Rényi entropy has been also used to explain dark energy [67].

Here it may be mentioned that both the Tsallis and Rényi entropy expressions belong to the one-parametric entropy family; there is also a two-parametric generalized entropy which is called Sharma-Mittal entropy $\left(S_{\mathrm{SM}}\right)$ and is written as $[67,69,70]$ :

$$
S_{\mathrm{SM}}=\frac{A_{0}}{G \alpha}\left\{\left(1+\frac{\delta}{4}\left(\frac{A}{A_{0}}\right)\right)^{\frac{\alpha}{\delta}}-1\right\},
$$

where $A_{0}$ is a constant, and $\alpha$ and $\delta$ are two independent parameters. Some cosmic applications of Sharma-Mittal entropy can be found in [69], where the Hubble horizon plays the role of the cut-off, and no mutual interaction between the cosmos components is taken into account.

Above considerations of different entropies eventually lead to different scenarios of holographic dark energy, which will be discussed in the following two sections.

\section{Dark Energy Corresponding to Tsallis, Rényi, and Sharma-Mittal Entropies}

We assume Friedmann-Lemaittre-Robertson-Walker (FLRW) space-time with a flat spacial component, whose metric is given by

$$
d s^{2}=-d t^{2}+a^{2}(t) \sum_{i=1,2,3}\left(d x^{i}\right)^{2} .
$$

Here $a(t)$ is called a scale factor.

If we define the Hubble rate $H$ by $H=\frac{\dot{a}}{a}$, the radius $r_{H}$ of the cosmological horizon is given by

$$
r_{H}=\frac{1}{H} .
$$

Then the entropy in the region inside the cosmological horizon could be given by the Bekenstein-Hawking relation [82] in Equation (1). On the other hand, the flux of the energy $E$ or the increase of the heat $Q$ in the region is given by

$$
d Q=-d E=-\frac{4 \pi}{3} r_{H}^{3} \dot{\rho} d t=-\frac{4 \pi}{3 H^{3}} \dot{\rho} d t=\frac{4 \pi}{H^{2}}(\rho+p) d t,
$$

where we use the conservation law: $0=\dot{\rho}+3 H(\rho+p)$. Then, by using the Hawking temperature [83]

$$
T=\frac{1}{2 \pi r_{H}}=\frac{H}{2 \pi},
$$

and the first law of thermodynamics $T d S=d Q$, one obtains $\dot{H}=-4 \pi G(\rho+p)$, and by integrating the expression, one obtains the first FLRW equation:

$$
H^{2}=\frac{8 \pi G}{3} \rho+\frac{\Lambda}{3}
$$

Here the cosmological constant $\Lambda$ appears as a constant of the integration. 
Instead of Bekenstein-Hawking entropy, Equation (1), we may use the non-extensive, Tsallis entropy [49-51,54] in Equation (2). Then by applying the first law of thermodynamics to the system, instead of $\dot{H}=-4 \pi G(\rho+p)$, one gets [55]:

$$
\delta\left(\frac{H^{2}}{H_{1}^{2}}\right)^{1-\delta} \dot{H}=-4 \pi G(\rho+p) ;
$$

upon integrating which, one gets:

$$
\frac{\delta}{2-\delta} H_{1}^{2}\left(\frac{H^{2}}{H_{1}^{2}}\right)^{2-\delta}=\frac{8 \pi G}{3} \rho+\frac{\Lambda}{3} .
$$

Here a constant $H_{1}$ is defined by $A_{0} \equiv \frac{4 \pi}{H_{1}^{2}}$. Then if we define the energy density $\rho_{\mathrm{T}}$ and the pressure $p_{\mathrm{T}}$ by

$$
\begin{gathered}
\rho_{\mathrm{T}}=\frac{3}{8 \pi G}\left(H^{2}-\frac{\delta}{2-\delta} H_{1}^{2}\left(\frac{H^{2}}{H_{1}^{2}}\right)^{2-\delta}\right), \\
p_{\mathrm{T}}=\frac{\dot{H}}{4 \pi G}\left\{\delta\left(\frac{H^{2}}{H_{1}^{2}}\right)^{1-\delta}-1\right\}-\frac{3}{8 \pi G}\left(H^{2}-\frac{\delta}{2-\delta} H_{1}^{2}\left(\frac{H^{2}}{H_{1}^{2}}\right)^{2-\delta}\right),
\end{gathered}
$$

respectively. It is evident that $\rho_{\mathrm{T}}$ depends on the quadratic power of the Hubble parameter and thus is symmetric with respect to the Hubble parameter. With the above forms of $\rho_{\mathrm{T}}$ and $p_{\mathrm{T}}$, Equations (10) and (11) can be expressed as

$$
\begin{aligned}
\dot{H} & =-4 \pi G\left[(\rho+p)+\left(\rho_{\mathrm{T}}+p_{\mathrm{T}}\right)\right], \\
H^{2} & =\frac{8 \pi G}{3}\left(\rho_{\mathrm{T}}+\rho\right)+\frac{\Lambda}{3},
\end{aligned}
$$

respectively. Therefore, $\rho_{\mathrm{T}}$ and $p_{\mathrm{T}}$ represent the energy density and pressure correspond ing to Tsallis entropy. Consequently, the respective equation of state (EoS) parameter for Tsallis entropy is given by

$$
\omega_{\mathrm{T}}=\frac{p_{\mathrm{T}}}{\rho_{\mathrm{T}}}=-1+2\left(\frac{\dot{H}}{H^{2}}\right)\left\{\frac{\delta\left(\frac{H^{2}}{H_{1}^{2}}\right)^{1-\delta}-1}{1-\frac{\delta}{2-\delta}\left(\frac{H^{2}}{H_{1}^{2}}\right)^{1-\delta}}\right\}
$$

It may be checked that the above expression of $\omega_{\mathrm{T}}$ leads to the conservation equation for the Tsallis entropic energy density-i.e.,

$$
\dot{\rho}_{\mathrm{T}}+3 H \rho_{\mathrm{T}}\left(1+\omega_{\mathrm{T}}\right)=0 .
$$

Here it deserves mentioning that the authors of [55] showed that the $\omega_{\mathrm{T}}$ in Equation (15) leads to a viable dark energy epoch of our present Universe, where the matter sector is considered to be dust. Moreover, the analysis is also extended to the case where the radiation energy density is present too. In particular, due to the Tsallis entropic energy density, the Universe exhibits the usual thermal history, with the sequence of matter and dark-energy eras and the onset of acceleration occurs at around $z \approx 0.5$ which is in agreement with observations [55]. 
In regard to the Rényi entropy Equation (3), the first law of thermodynamics gives

$$
-\frac{H^{3} \dot{H}}{H^{2}+\frac{\delta}{4} H_{1}^{2}}=-\frac{4 \pi G}{3} \dot{\rho},
$$

from which, we obtain

$$
H^{2}=\frac{8 \pi G}{3} \rho+\frac{\Lambda}{3}+\frac{\delta}{4} H_{1}^{2} \ln \left(\frac{H^{2}}{H_{1}^{2}}+\frac{\delta}{4}\right) .
$$

Here the cosmological constant $\Lambda$ appears as a constant of the integration again. At this stage we may define the corresponding energy density and the pressure in the following form:

$$
\begin{gathered}
\rho_{\mathrm{R}}=\frac{3 \delta}{32 G} H_{1}^{2} \ln \left(\frac{H^{2}}{H_{1}^{2}}+\frac{\delta}{4}\right), \\
p_{\mathrm{R}}=-\frac{\dot{H}}{4 \pi G}\left\{\frac{1}{1+\frac{4}{\delta}\left(\frac{H^{2}}{H_{1}^{2}}\right)}\right\}-\frac{3 \delta}{32 G} H_{1}^{2} \ln \left(\frac{H^{2}}{H_{1}^{2}}+\frac{\delta}{4}\right) .
\end{gathered}
$$

Similarly to the Tsallis entropic case, the Rényi entropic energy density (i.e., $\rho_{R}$ ) seems to be symmetric with respect to the Hubble parameter. Due to the above expressions of $\rho_{\mathrm{R}}$ and $p_{\mathrm{R}}$, Equations (17) and (18) become similar to the usual Friedmann equations where the total energy density and total pressure are given by $\rho_{\text {eff }}=\rho+\rho_{\mathrm{R}}$ and $p_{\text {eff }}=$ $p+p_{\mathrm{R}}$. Consequently, the EoS parameter corresponds to the Rényi entropy, and takes the following form:

$$
\omega_{\mathrm{R}}=\frac{p_{\mathrm{R}}}{\rho_{\mathrm{R}}}=-1-\frac{8}{3 \pi \delta}\left(\frac{\dot{H}}{H_{1}^{2}}\right)\left\{\frac{1}{\ln \left(\frac{H^{2}}{H_{1}^{2}}+\frac{\delta}{4}\right)\left[1+\frac{4}{\delta}\left(\frac{H^{2}}{H_{1}^{2}}\right)\right]}\right\} .
$$

It may be mentioned that the above expression of $\omega_{\mathrm{R}}$ obeys the conservation equation for Rényi entropic energy density. As shown in $[67,68]$, the Rényi entropic energy density $\left(\rho_{R}\right)$ and the pressure $\left(p_{R}\right)$ can provide suitable description for the current accelerated Universe, thereby leading to a dark energy model.

In case of Sharma-Mittal entropy, the first law of thermodynamics leads to the following evolution of the cosmic Hubble parameter:

$$
\left(1+\frac{\delta H_{1}^{2}}{4 H^{2}}\right)^{\frac{\alpha}{\delta}-1} \dot{H}=-4 \pi G(\rho+p),
$$

by integrating which, we obtain

$$
H_{1}^{2}\left(\frac{\left(\frac{\delta}{4}\right)^{\frac{\alpha}{\delta}-1}}{2-\alpha / \delta}\right)\left(\frac{H^{2}}{H_{1}^{2}}\right)^{2-\frac{\alpha}{\delta}}{ }_{2} F_{1}\left[1-\frac{\alpha}{\delta}, 2-\frac{\alpha}{\delta}, 3-\frac{\alpha}{\delta} ;-\frac{4}{\delta}\left(\frac{H^{2}}{H_{1}^{2}}\right)\right]=\frac{8 \pi G}{3} \rho+\frac{\Lambda}{3},
$$

where $\Lambda$ is the constant of integration, ${ }_{2} F_{1}$ is the hypergeometric function, and to get the above expression, we use the conservation equation of the matter components. Moreover, 
the constant $H_{1}$ is related to $A_{0}$ by $A_{0}=\frac{4 \pi}{H_{1}^{2}}$. Now if we define an energy density $\left(\rho_{\mathrm{SM}}\right)$ and a pressure $\left(p_{\mathrm{SM}}\right)$ like

$$
\begin{gathered}
\rho_{\mathrm{SM}}=\frac{3}{8 \pi G}\left\{H^{2}-H_{1}^{2}\left(\frac{\left(\frac{\delta}{4}\right)^{\frac{\alpha}{\delta}-1}}{2-\frac{\alpha}{\delta}}\right)\left(\frac{H^{2}}{H_{1}^{2}}\right)^{2-\frac{\alpha}{\delta}}{ }_{2} F_{1}\left[1-\frac{\alpha}{\delta}, 2-\frac{\alpha}{\delta}, 3-\frac{\alpha}{\delta} ;-\frac{4}{\delta}\left(\frac{H^{2}}{H_{1}^{2}}\right)\right]\right\}, \\
p_{\mathrm{SM}}=\frac{\dot{H}}{4 \pi G}\left\{\left(1+\frac{\delta H_{1}^{2}}{4 H^{2}}\right)^{\frac{\alpha}{\delta}-1}-1\right\}-\rho_{\mathrm{SM}},
\end{gathered}
$$

respectively, then Equations (22) and (23) can be equivalently expressed as

$$
\begin{aligned}
\dot{H} & =-4 \pi G\left[(\rho+p)+\left(\rho_{\mathrm{SM}}+p_{\mathrm{SM}}\right)\right], \\
H^{2} & =\frac{8 \pi G}{3}\left(\rho_{\mathrm{SM}}+\rho\right)+\frac{\Lambda}{3} .
\end{aligned}
$$

Thus we may argue that $\rho_{\mathrm{SM}}$ and $p_{\mathrm{SM}}$ are the energy density and the pressure coming from the cosmological description of Sharma-Mittal entropy. Furthermore, $\rho_{\mathrm{SM}}$ and $p_{\mathrm{SM}}$ are connected by the respective EoS, as given by

$$
\omega_{\mathrm{SM}}=-1+\left(\frac{\dot{H}}{3 H^{2}}\right)\left\{\frac{\left(1+\frac{\delta H_{1}^{2}}{4 H^{2}}\right)^{\frac{\alpha}{\delta}-1}-1}{1-\left(\frac{\left(\frac{\delta}{4} \frac{\alpha}{2}-\frac{\alpha}{\delta}\right.}{\delta}\right)\left(\frac{H_{1}^{2}}{H^{2}}\right)^{\frac{\alpha}{\delta}-1}{ }_{2} F_{1}\left[1-\frac{\alpha}{\delta}, 2-\frac{\alpha}{\delta}, 3-\frac{\alpha}{\delta} ;-\frac{4}{\delta}\left(\frac{H^{2}}{H_{1}^{2}}\right)\right]}\right\},
$$

where we use Equations (24) and (25). The above form of $\omega_{\mathrm{SM}}$ immediately confirms the conservation equation for the Sharma-Mittal entropic energy density. Furthermore, as established in $[67,69]$, the Sharma-Mittal entropic energy density leads to a late-time acceleration epoch of our Universe. In [69], the Universe was considered to be filled by a pressureless component and Sharma-Mittal entropic energy density, which do not have any mutual interaction, and as a result, the present deceleration parameter was found to be consistent with the present observation.

Before closing this section, here we would like to mention that the presence of entropic energy densities indeed modify the FLRW equations. Such modifications can also be encapsulated in the respective entropy functions. In particular, when we consider the Bekenstein-Hawking entropy in the context of cosmology, one gets the usual FLRW equations and thus we have the expression like $\frac{d S}{d H}=-2 \pi /\left(G H^{3}\right)$ which leads to $S=S(H)$. However for the modified entropy cases, the relation of $S=S(H)$ will become different compared to the Bekenstein-Hawking case. In particular, for the Tsallis entropy case, the first FLRW Equation (11) leads to the following expression:

$$
\delta\left(\frac{H^{2}}{H_{1}^{2}}\right)^{1-\delta} H d H=\frac{4 \pi G}{3} d \rho,
$$

which along with the first law of thermodynamics (in Equation (7)), yields the respective entropy in terms of the Hubble parameter as

$$
\frac{d S_{\mathrm{T}}}{d H}=-\frac{2 \pi \delta}{G}\left(\frac{1}{H^{3}}\right)\left(\frac{H^{2}}{H_{1}^{2}}\right)^{1-\delta},
$$

which on integration, becomes $S_{\mathrm{T}}=S_{\mathrm{T}}(H)$. It is evident that for $\delta=1$, the above expression becomes similar to that of the Bekenstein-Hawking case. Thereby the modification of the Tsallis entropy compared to the Bekenstein-hawking case is clearly demonstrated by 
the expression of $S_{\mathrm{T}}=S_{\mathrm{T}}(H)$. By a similar procedure, we can obtain the Rényi entropy and the Sharma-Mittal entropy functions in terms of the Hubble parameter as

$$
\begin{aligned}
\frac{d S_{\mathrm{R}}}{d H} & =-\frac{2 \pi}{G}\left(\frac{1}{H^{3}}\right) \frac{1}{\left(1+\frac{\delta}{4}\left(\frac{H_{1}^{2}}{H^{2}}\right)\right)}, \\
\frac{d S_{\mathrm{SM}}}{d H} & =-\frac{2 \pi}{G}\left(\frac{1}{H^{3}}\right)\left(1+\frac{\delta}{4}\left(\frac{H_{1}^{2}}{H^{2}}\right)\right)^{\frac{\alpha}{\delta}-1} .
\end{aligned}
$$

Clearly, Equation (30) depicts that for $\delta=1$, one gets $\frac{d S_{\mathrm{R}}}{d H}=-2 \pi /\left(G H^{3}\right)$, while Equation (31) reveals that the situation $\alpha=\delta$ leads to $\frac{d S_{S M}}{d H}=-2 \pi /\left(G H^{3}\right)$; i.e., they become similar to that of the Bekenstein-Hawking entropy function for the aforesaid conditions, respectively. Here we would like to mention that Equations (29)-(31) remains symmetric under the transformation $H \rightarrow-H$.

\section{Generalized Holographic Energy}

In the holographic principle, the holographic energy density is proportional to the inverse squared infrared cutoff $L_{\mathrm{IR}}$, which could be related to the causality given by the cosmological horizon:

$$
\rho_{\text {hol }}=\frac{3 c^{2}}{\kappa^{2} L_{\mathrm{IR}}^{2}} .
$$

Here $\kappa^{2}=8 \pi G$ is the gravitational constant and $c$ is a free parameter. The infrared cutoff $L_{\mathrm{IR}}$ is usually assumed to be the particle horizon $L_{\mathrm{p}}$ or the future event horizon $L_{\mathrm{f}}$, which are given as

$$
L_{\mathrm{p}} \equiv a \int_{0}^{t} \frac{d t}{a}, \quad L_{\mathrm{f}} \equiv a \int_{t}^{\infty} \frac{d t}{a} .
$$

Differentiating both sides of the above expressions leads to the Hubble parameter in terms of $L_{\mathrm{p}}, \dot{L}_{\mathrm{p}}$ or in terms of $L_{\mathrm{f}}, \dot{L}_{\mathrm{f}}$ as

$$
H\left(L_{\mathrm{p}}, \dot{L}_{\mathrm{p}}\right)=\frac{\dot{L}_{\mathrm{p}}}{L_{\mathrm{p}}}-\frac{1}{L_{\mathrm{p}}}, \quad H\left(L_{\mathrm{f}}, \dot{L}_{\mathrm{f}}\right)=\frac{\dot{L}_{\mathrm{f}}}{L_{\mathrm{f}}}+\frac{1}{L_{\mathrm{f}}} .
$$

In [9], a general form of the cutoff was proposed:

$$
L_{\mathrm{IR}}=L_{\mathrm{IR}}\left(L_{\mathrm{p}}, \dot{L}_{\mathrm{p}}, \ddot{L}_{\mathrm{p}}, \ldots, L_{\mathrm{f}}, \dot{L}_{\mathrm{f}}, \ldots, a\right) .
$$

Actually, the other dependency of $L_{\mathrm{IR}}$, particularly on the Hubble parameter, the Ricci scalar and their derivatives, can be transformed to either $L_{p}$ and their derivatives or $L_{f}$ and their derivatives via Equation (34). The above cutoff could be chosen to be equivalent to a general covariant gravity model:

$$
S=\int d^{4} \sqrt{-g} F\left(R, R_{\mu v} R^{\mu v}, R_{\mu v \rho \sigma} R^{\mu \nu \rho \sigma}, \square R, \square^{-1} R, \nabla_{\mu} R \nabla^{\mu} R, \ldots\right) .
$$

We use the above expressions frequently in the following sections. With the help of the generalized cut-off, we aim to show that the Tsallis, Rényi, and Sharma-Mittal entropic dark energy may belong from the generalized dark energy family where the holographic cut-offs are expressed in terms of the particle horizon and its derivatives or in terms of the future horizon and its derivatives. 
A comparison of Equations (12) and (32) leads to the argument that Tsallis entropic dark energy belongs to the generalized holographic dark energy family, where the corresponding infrared cutoff $L_{\mathrm{T}}$ is given by

$$
\frac{3 c^{2}}{\kappa^{2} L_{\mathrm{T}}^{2}}=\frac{3}{8 \pi G}\left(\left(\frac{\dot{L}_{\mathrm{p}}}{L_{\mathrm{p}}}-\frac{1}{L_{\mathrm{p}}}\right)^{2}-\frac{\delta}{2-\delta} H_{1}^{2}\left(\frac{\left(\frac{\dot{L}_{\mathrm{p}}}{L_{\mathrm{p}}}-\frac{1}{L_{\mathrm{p}}}\right)^{2}}{H_{1}^{2}}\right)^{2-\delta}\right),
$$

in terms of $L_{\mathrm{p}}$ and its derivatives. To get the above expression, we use Equation (34). Moreover, $L_{\mathrm{T}}$ in terms of the future horizon and its derivatives are as follows:

$$
\frac{3 c^{2}}{\kappa^{2} L_{\mathrm{T}}^{2}}=\frac{3}{8 \pi G}\left(\left(\frac{\dot{L}_{\mathrm{f}}}{L_{\mathrm{f}}}+\frac{1}{L_{\mathrm{f}}}\right)^{2}-\frac{\delta}{2-\delta} H_{1}^{2}\left(\frac{\left(\frac{\dot{L}_{\mathrm{f}}}{L_{\mathrm{f}}}+\frac{1}{L_{\mathrm{f}}}\right)^{2}}{H_{1}^{2}}\right)^{2-\delta}\right) .
$$

Here we would like to determine whether the EoS parameter of the holographic energy density corresponds to the cut-off $L_{\mathrm{T}}$, in particular, of $\rho_{\text {hol }}=3 c^{2} /\left(\kappa^{2} L_{\mathrm{T}}^{2}\right)$. In this regard, the conservation equation of $\rho_{\text {hol }}$ immediately yields the respective EoS parameter (symbolized by $\Omega_{\text {hol }}^{(T)}$ ) as

$$
\Omega_{\mathrm{hol}}^{(T)}=-1-\left(\frac{2}{3 H L_{\mathrm{T}}}\right) \frac{d L_{\mathrm{T}}}{d t},
$$

where $L_{\mathrm{T}}$ is obtained in Equation (37) (or Equation (38)) and the superscript " $\mathrm{T}$ " in the above expression denotes the EoS parameter corresponds to the holographic cut-off $L_{\mathrm{T}}$. Due to Equation (34), the above form of $\Omega_{\text {hol }}^{(T)}$ seems to be equivalent to the EoS of the Tsallis entropic energy density presented in Equation (15), i.e., $\Omega_{\text {hol }}^{(T)} \equiv \omega_{\mathrm{T}}$. Said equivalence, along with the fact that the Tsallis entropic energy density provides a viable dark energy model, lead to the argument that the holographic energy density coming from the cut-off $L_{\mathrm{T}}$ is also able to produce a viable dark energy epoch at our current Universe.

Similarly, by comparing Equations (19) and (32), the infrared cutoff $L_{R}$ corresponding to the Rényi entropy is given by

$$
\frac{3 c^{2}}{\kappa^{2} L_{\mathrm{R}}^{2}}=\frac{3 \delta}{32 G} H_{1}^{2} \ln \left(\frac{1}{H_{1}^{2}}\left(\frac{\dot{L}_{\mathrm{p}}}{L_{\mathrm{p}}}-\frac{1}{L_{\mathrm{p}}}\right)^{2}+\frac{\delta}{4}\right)=\frac{3 \delta}{32 G} H_{1}^{2} \ln \left(\frac{1}{H_{1}^{2}}\left(\frac{\dot{L}_{\mathrm{f}}}{L_{\mathrm{f}}}+\frac{1}{L_{\mathrm{f}}}\right)^{2}+\frac{\delta}{4}\right),
$$

where, once again, we used Equation (34). The first expression of Equation (40) gives the $L_{R}$ in terms of $L_{\mathrm{p}}$ and its derivatives, while the second one represents the same in terms of $L_{\mathrm{f}}$ and its derivatives. Once again, the conservation equation of the holographic energy density $\rho_{\text {hol }}=3 c^{2} /\left(\kappa^{2} L_{\mathrm{R}}^{2}\right)$ leads to the corresponding EoS parameter $\left(\Omega_{\mathrm{hol}}^{(R)}\right)$ as

$$
\Omega_{\mathrm{hol}}^{(R)}=-1-\left(\frac{2}{3 H L_{\mathrm{R}}}\right) \frac{d L_{\mathrm{R}}}{d t},
$$

where $L_{\mathrm{R}}$ is given in Equation (40). It can be easily checked that the $\Omega_{\text {hol }}^{(R)}$ satisfies the conservation relation: $\dot{\rho}_{\text {hol }}+3 H\left(1+\Omega_{\text {hol }}^{(R)}\right)=0$, where $\rho_{\text {hol }}$ represents the holographic energy density coming from the cut-off $L_{R}$. Equations (34) and (40) indicate that the above expression of $\Omega_{\text {hol }}^{(R)}$ proves to be equivalent with $\omega_{\mathrm{R}}$ in Equation (21), i.e., $\Omega_{\mathrm{hol}}^{(R)} \equiv \omega_{\mathrm{R}}$. Thereby, since the Rényi entropic energy density suitably describes the current acceleration of our Universe, we may argue that the holographic energy density coming from $L_{R}$ is able to produce the late time cosmic acceleration. 
Finally, Equations (24) and (32) clearly argue that Sharma-Mittal entropic dark energy can also be thought as one of the candidates for the generalized dark energy family, where the corresponding cut-off $\left(L_{\mathrm{SM}}\right)$ is given by

$$
\begin{aligned}
\frac{3 c^{2}}{\kappa^{2} L_{\mathrm{SM}}^{2}}=\frac{3}{8 \pi G}\left\{\left(\frac{\dot{L}_{\mathrm{p}}}{L_{\mathrm{p}}}-\frac{1}{L_{\mathrm{p}}}\right)^{2}-H_{1}^{2}\left(\frac{\left(\frac{\delta}{4}\right)^{\frac{\alpha}{\delta}-1}}{2-\alpha / \delta}\right)\left(\frac{\left(\frac{\dot{L}_{\mathrm{p}}}{L_{\mathrm{p}}}-\frac{1}{L_{\mathrm{p}}}\right)^{2}}{H_{1}^{2}}\right)^{2-\frac{\alpha}{\delta}}\right. \\
\\
\left.\quad{ }_{2} F_{1}\left[1-\frac{\alpha}{\delta}, 2-\frac{\alpha}{\delta}, 3-\frac{\alpha}{\delta} ;-\frac{4}{\delta}\left(\frac{\left(\frac{\dot{L}_{\mathrm{p}}}{L_{\mathrm{p}}}-\frac{1}{L_{\mathrm{p}}}\right)^{2}}{H_{1}^{2}}\right)\right]\right\},
\end{aligned}
$$

in terms of the particle horizon and its derivatives. Similarly, the $L_{S M}$ in terms of the future horizon and its derivatives is given by

$$
\begin{aligned}
\frac{3 c^{2}}{\kappa^{2} L_{\mathrm{SM}}^{2}}=\frac{3}{8 \pi G}\left\{\left(\frac{\dot{L}_{\mathrm{f}}}{L_{\mathrm{f}}}+\frac{1}{L_{\mathrm{f}}}\right)^{2}-H_{1}^{2}\left(\frac{\left(\frac{\delta}{4}\right)^{\frac{\alpha}{\delta}-1}}{2-\frac{\alpha}{\delta}}\right)\left(\frac{\left(\frac{\dot{L}_{\mathrm{f}}}{L_{\mathrm{f}}}+\frac{1}{L_{\mathrm{f}}}\right)^{2}}{H_{1}^{2}}\right)^{2-\frac{\alpha}{\delta}}\right. \\
\\
\left.\quad{ }_{2} F_{1}\left[1-\frac{\alpha}{\delta}, 2-\frac{\alpha}{\delta}, 3-\frac{\alpha}{\delta} ;-\frac{4}{\delta}\left(\frac{\left(\frac{\dot{L}_{\mathrm{f}}}{L_{\mathrm{f}}}+\frac{1}{L_{\mathrm{f}}}\right)^{2}}{H_{1}^{2}}\right)\right]\right\} .
\end{aligned}
$$

Furthermore, using the conservation relation of $\rho_{\text {hol }}=3 c^{2} /\left(\kappa^{2} L_{\mathrm{SM}}^{2}\right)$, we can determine that the EoS parameter $\left(\Omega_{\mathrm{hol}}^{(S M)}\right)$ corresponds to the holographic energy density coming from the cut-off $L_{\mathrm{SM}}$ as

$$
\Omega_{\mathrm{hol}}^{(S M)}=-1-\left(\frac{2}{3 H L_{\mathrm{SM}}}\right) \frac{d L_{\mathrm{SM}}}{d t},
$$

where $L_{S M}$ is given in Equation (42) (or in Equation (43)). Due to Equation (34), it is evident that the above form of $\Omega_{\text {hol }}^{(S M)}$ is equivalent to the EoS of the Sharma-Mittal entropic energy density of Equation (27), i.e., $\Omega_{\text {hol }}^{(S M)}=\omega_{S M}$. Due to this equivalence, we may argue that the holographic energy density $\rho_{\text {hol }}=3 c^{2} /\left(\kappa^{2} L_{\mathrm{SM}}^{2}\right)$ can produce the late-time acceleration of our Universe.

Therefore, the dark energy models coming from Tsallis entropy, the Rényi entropy, and Sharma-Mittal entropy can be thought as different candidates for the generalized holographic dark energy family, and the respective infrared cutoffs are given by Equations (37)-(43) respectively. Thereby, such holographic cut-offs establish symmetry between generalized HDE and the respective entropic DE model(s).

\section{Extended Cases of Entropic Dark Energy Models}

In this section we consider the models extended as in [86], where the non-extensive exponent $\delta$ of Tsallis entropy, Equation (2), or Rényi entropy Equation (3), depends on the energy scale and shows a running behavior [87]. In [87], it has been claimed that such behaviors may appear because the entropy corresponds to physical degrees of freedom and the degrees of freedom depend on the scale, as implied by the renormalization of a quantum theory. In the case of gravity, if the space-time fluctuates at high energy scales, the degrees of freedom may increase. On the other hand, if gravity becomes a topological theory, the degrees of freedom may decrease.

In cosmology, if we assume that the energy scale could be given by the Hubble scale $H$, $\delta$ in Equation (2), or Equation (3) may depend on $H$ [87]. Thereby we use a dimensionless variable $x \equiv \frac{H_{1}^{2}}{H^{2}}$ by introducing a parameter $H_{1}$ whose dimension is identical to $H$. Then in 
case of the Tsallis entropy Equation (2), instead of Equation (11), one obtains the following generalized first FLRW equation (see the Appendix A for the detailed derivation),

$$
-\left.H_{1}^{2}\left\{x^{\delta(x)-2}+2 \int^{x} d x x^{\delta(x)-3}\right\}\right|_{x=\frac{H_{1}^{2}}{H^{2}}}=\frac{8 \pi G}{3} \rho+\frac{\Lambda}{3}
$$

and we may define the effective energy density $\rho_{\mathrm{T}}$ by

$$
\rho_{\mathrm{T}} \equiv \frac{3}{8 \pi G}\left(H^{2}+\left.H_{1}^{2}\left\{x^{\delta(x)-2}+2 \int^{x} d x x^{\delta(x)-3}\right\}\right|_{x=\frac{H_{1}^{2}}{H^{2}}}\right)
$$

Thereby the presence of a varying exponent in the Tsallis entropy modifies the FLRW equations, which may have considerable impacts in the Universe's evolution, both at high and low energy scales. Clearly, in order to determine the explicit expression of $\rho_{\mathrm{T}}$ from the above equation, one needs a functional form of $\delta(x)$. Some of our authors proposed a suitable form of $\delta(x)$ in [87], which allows one to analytically perform the integration in Equation (46) and also leads to an unified scenario of early inflation with late-time acceleration. Actually, the form of $\delta(x)$ was chosen in such a way that at high and low energy scales it acquires values away from the standard value 1 , and at intermediate scales it comes close to unity [87].

In case of the Rényi entropy Equation (3), the first law of thermodynamics gives (see the Appendix A for the detailed derivation)

$$
H^{2}=\frac{8 \pi G}{3} \rho+\frac{\Lambda}{3}+\left.4 H_{1}^{2} \int^{x} d x x^{-2}\left\{\left(\frac{\frac{\delta(x)}{16}}{1+\frac{\delta(x)}{4} x}\right)+\left(\frac{1}{\delta(x)^{2}} \ln \left(1+\frac{\delta(x)}{4} x\right)-\frac{1}{\delta(x)} \frac{\frac{1}{4} x}{1+\frac{\delta(x)}{4} x}\right) \delta^{\prime}(x)\right\}\right|_{x=\frac{H_{1}^{2}}{H^{2}}}
$$

where $\delta^{\prime}(x)=\frac{d \delta}{d x}$. Then we may define the effective energy density $\rho_{\mathrm{R}}$ corresponding to Rényi entropy by

$$
\left.\rho_{\mathrm{R}} \equiv 4 H_{1}^{2} \int^{x} d x x^{-2}\left\{\left(\frac{\frac{\delta(x)}{16}}{1+\frac{\delta(x)}{4} x}\right)+\left(\frac{1}{\delta(x)^{2}} \ln \left(1+\frac{\delta(x)}{4} x\right)-\frac{1}{\delta(x)} \frac{\frac{1}{4} x}{1+\frac{\delta(x)}{4} x}\right) \delta^{\prime}(x)\right\}\right|_{x=\frac{H_{1}^{2}}{H^{2}}} .
$$

The terms containing $\delta^{\prime}(x)$ in the above expression of $\rho_{\mathrm{R}}$ arise due to the varying exponent $\delta=\delta(x)$. Such terms may play an important role during the early and late cosmic evolution of the Universe. In particular, we expect that at high and low energy scales, the $\delta(x)$ should deviate from the standard value unity and thus have a significant role in driving the inflation or the late dark energy epoch. However the modified cosmology from the Rényi entropy with a varying exponent has not been extensively studied in various earlier literature. Thereby, it will be an interesting avenue to study the possible effects of $\rho_{R}$ in Equation (48) in the context of inflation or late-time acceleration, or even in the bouncing scenario. However these are out of the scope from the present work, and thus we expect to study it in future work.

Coming back to Sharma-Mittal entropy, there are two independent parameters ( $\alpha$ and $\delta$ ), and in the extended scenario, we take $\alpha=\alpha(x)$ and $\delta$ to be constant. However, in the extended case of the Sharma-Mittal entropy, one may choose both the parameters being dependent on $x=H_{1}^{2} / H^{2}$, i.e., $\alpha=\alpha(x)$ and $\delta=\delta(x)$. For simplicity, here we stick to the aforementioned consideration, i.e., $\alpha=\alpha(x)$ and $\delta=$ constant. Said consideration leads to the FLRW equation as (see the Appendix A for the detailed derivation)

$$
-\left.H_{1}^{2} f(x)\right|_{x=\frac{H_{1}^{2}}{H^{2}}}=\frac{8 \pi G}{3} \rho+\frac{\Lambda}{3},
$$

with, 
$f(x)=\int^{x} d x x^{-2}\left\{\left(1+\frac{\delta x}{4}\right)^{\frac{\alpha(x)}{\delta}-1}-\frac{4 \alpha^{\prime}(x)}{\alpha(x)}\left[\left(1+\frac{\delta x}{4}\right)^{\frac{\alpha(x)}{\delta}}-1\right]+\frac{4 \alpha^{\prime}(x)}{\alpha(x) \delta}\left(1+\frac{\delta x}{4}\right)^{\frac{\alpha(x)}{\delta}} \ln \left(1+\frac{\delta x}{4}\right)\right\}$.

Consequently, the effective energy density corresponding to Sharma-Mittal entropy can be shown as follows.

$$
\rho_{\mathrm{SM}}=\left.\frac{3}{8 \pi G}\left\{H^{2}+H_{1}^{2} f(x)\right\}\right|_{x=\frac{H_{1}^{2}}{H^{2}}} .
$$

Equations (46), (48), and (51) immediately tell one that the infrared cut-offs $L_{T}, L_{R}$, and $L_{\mathrm{SM}}$ corresponding to the extended versions of Tsallis entropy, Rényi entropy, and Sharma-Mittal entropy are given by

$$
\begin{array}{r}
\frac{3 c^{2}}{\kappa^{2} L_{\mathrm{T}}^{2}}=\frac{3}{8 \pi G}\left(\left(\frac{\dot{L}_{\mathrm{p}}}{L_{\mathrm{p}}}-\frac{1}{L_{\mathrm{p}}}\right)^{2}+\left.H_{1}^{2}\left\{x^{\delta(x)-2}+2 \int^{x} d x x^{\delta(x)-3}\right\}\right|_{\left.x=H_{1}^{2}\left(\frac{L_{\mathrm{p}}}{L_{\mathrm{p}}}-\frac{1}{L_{\mathrm{p}}}\right)^{-2}\right)}\right. \\
=\frac{3}{8 \pi G}\left(\left(\frac{\dot{L}_{\mathrm{f}}}{L_{\mathrm{f}}}+\frac{1}{L_{\mathrm{f}}}\right)^{2}+\left.H_{1}^{2}\left\{x^{\delta(x)-2}+2 \int^{x} d x x^{\delta(x)-3}\right\}\right|_{\left.x=H_{1}^{2}\left(\frac{L_{\mathrm{f}}}{L_{\mathrm{f}}}+\frac{1}{L_{\mathrm{f}}}\right)^{-2}\right),}\right. \\
\frac{3 c^{2}}{\mathcal{K}^{2} L_{\mathrm{R}}^{2}}=\left.\frac{4 H_{1}^{2}}{G} \int^{x} d x x^{-2}\left\{\left(\frac{\frac{\delta(x)}{16}}{1+\frac{\delta(x)}{4} x}\right)+\left(\frac{1}{\delta(x)^{2}} \ln \left(1+\frac{\delta(x)}{4} x\right)-\frac{1}{\delta(x)} \frac{\frac{1}{4} x}{1+\frac{\delta(x)}{4} x}\right) \delta^{\prime}(x)\right\}\right|_{x=H_{1}^{2}\left(\frac{L_{\mathrm{p}}}{L_{\mathrm{p}}}-\frac{1}{L_{\mathrm{p}}}\right)^{-2}} \\
=\left.\frac{4 H_{1}^{2}}{G} \int^{x} d x x^{-2}\left\{\left(\frac{\frac{\delta(x)}{16}}{1+\frac{\delta(x)}{4} x}\right)+\left(\frac{1}{\delta(x)^{2}} \ln \left(1+\frac{\delta(x)}{4} x\right)-\frac{1}{\delta(x)} \frac{\frac{1}{4} x}{1+\frac{\delta(x)}{4} x}\right) \delta^{\prime}(x)\right\}\right|_{x=H_{1}^{2}\left(\frac{L_{\mathrm{f}}}{L_{\mathrm{f}}}+\frac{1}{L_{\mathrm{f}}}\right)^{-2}}
\end{array}
$$

and

$$
\begin{aligned}
\frac{3 c^{2}}{\kappa^{2} L_{\mathrm{SM}}^{2}} & =\left.\frac{3}{8 \pi G}\left\{\left(\frac{\dot{L}_{\mathrm{p}}}{L_{\mathrm{p}}}-\frac{1}{L_{\mathrm{p}}}\right)^{2}+H_{1}^{2} f(x)\right\}\right|_{x=H_{1}^{2}\left(\frac{\dot{L}_{\mathrm{p}}}{L_{\mathrm{p}}}-\frac{1}{L_{\mathrm{p}}}\right)^{-2}} \\
& =\left.\frac{3}{8 \pi G}\left\{\left(\frac{\dot{L}_{\mathrm{f}}}{L_{\mathrm{f}}}+\frac{1}{L_{\mathrm{f}}}\right)^{2}+H_{1}^{2} f(x)\right\}\right|_{x=H_{1}^{2}\left(\frac{\dot{L}_{\mathrm{f}}}{L_{\mathrm{f}}}+\frac{1}{L_{\mathrm{f}}}\right)^{-2}}
\end{aligned}
$$

respectively, with $f(x)$ being shown in Equation (50). Therefore, even for the extended case, the holographic energies coming from Tsallis entropy, Rényi entropy, and SharmaMittal entropy can be expressed by the general infrared cutoffs in [9]. Interestingly, the corresponding cut-offs are determined in terms of the particle horizon and its derivative or in terms of the future horizon and its derivative.

\section{Some Other DE Models and Their Equivalence with Generalized HDE}

Besides the Tsallis, Rényi, and Sharma-Mittal entropic DE scenario, some other DE models, in particular the quintessence [71-74] and Ricci DE models [23,29,76], are included the present analysis. Their equivalence with the generalized HDE and the corresponding cut-offs are discussed in the following two subsections respectively.

\subsection{Quintessence Dark Energy}

The present observation indicates that the equation of state parameter at the present Universe is close to $\omega \simeq-1$; however, this says little about the time evolution of $\omega$, and 
thus we can broaden our situation and consider a dark energy model where the equation of state changes with time. Said dark energy models are the scalar field dark energy models where the dynamics of the scalar field over the FLRW space-time leads to a evolving EoS of the Universe. So far, a large number of scalar field dark energy models have been proposed, these include quintessence, phantoms, k-essence, tachyon, and dilatonic dark energy.

In this section, we consider the quintessence dark energy (QDE) model and aim to show that QDE is equivalent to the generalized holographic dark energy model, where $L_{\mathrm{IR}}=L_{\mathrm{IR}}\left(L_{\mathrm{p}}, \dot{L}_{\mathrm{p}}, \ddot{L}_{\mathrm{p}}, L_{\mathrm{f}}, \dot{L}_{\mathrm{f}}, \ddot{L}_{\mathrm{f}}\right)$. The QDE action is given by

$$
S=\int d^{4} x \sqrt{-g}\left[\frac{R}{16 \pi G}-\frac{1}{2} g^{\mu \nu} \partial_{\mu} \phi \partial_{\nu} \phi-V(\phi)\right],
$$

where $\phi$ is the quintessence scalar field and $V(\phi)$ is its potential. The presence of the potential is important in the dark energy context; otherwise the energy density and pressure of the scalar field become equal, which in turn leads to a decelerating expansion of the Universe. In particular, with $V(\phi)=0$, the FLRW scale factor of the Universe evolves as $a(t) \sim t^{2 / 3}$, and thus the scalar field model without potential is not compatible with dark energy observations. The quintessence potential has the following form [71-74],

$$
V(\phi)=V_{0} \exp \left[-\sqrt{\frac{16 \pi G}{p}} \phi\right],
$$

with $V_{0}$ and $p$ being constants. The quintessence model with the above exponential potential has been extensively studied in [74], where it was shown that the potential of Equation (56) leads to a viable dark energy model with respect to SNIa, BAO, and H(z) observations. However, the most stringent constraint on the dark energy EoS parameter $\left(\omega_{\mathrm{Q}}\right)$ comes from the BAO observations-in particular, $-1<\omega_{\mathrm{Q}}<-0.85$ [74].

The FLRW equations corresponding to the action Equation (55) are

$$
\begin{aligned}
H^{2} & =\frac{8 \pi G}{3}\left(\frac{1}{2} \dot{\phi}^{2}+V(\phi)\right), \\
\dot{H} & =-4 \pi G \dot{\phi}^{2},
\end{aligned}
$$

where, due to the homogeneity, the scalar field is considered to be the function of time only. The first FLRW equation immediately leads to the quintessence energy density as

$$
\rho_{\mathrm{Q}}=\frac{1}{2} \dot{\phi}^{2}+V(\phi)=-\frac{\dot{H}}{8 \pi G}+V(\phi) \text {, }
$$

where in the second line, we use $\dot{H}=-4 \pi G \dot{\phi}^{2}$. Equation (58) shows that the quintessence energy density is not symmetric with respect to the Hubble parameter, unlike the case of entropic dark energy models (that we considered earlier) where the entropic energy density proves to be symmetric with respect to the Hubble parameter. The exponential form of the quintessence potential (see Equation (56)) allows the following solutions of the Hubble parameter and the scalar field:

$$
H=\frac{p}{t} \quad \text { and } \quad \phi(t)=2 \sqrt{\frac{p}{16 \pi G}} \ln \left(\frac{t}{t_{0}}\right),
$$

respectively. Here $t_{0}$ is fudicial time and $V_{0}, p$, and $t_{0}$ are related by the following constraint equation:

$$
3 p-1=V_{0} t_{0}^{2}\left(\frac{8 \pi G}{p}\right)
$$


Furthermore, the evolution of the Hubble parameter clearly indicates that in order to get an accelerating expansion of the Universe, the parameter $p$ is constrained to be $p>1$. By using Equations (56) and (59), we can express the quintessence potential in terms of the Hubble parameter as follows:

$$
V(\phi)=\left(3-\frac{1}{p}\right) \frac{H^{2}}{8 \pi G} .
$$
$\dot{H}$ as

Plugging back the above expression into Equation (58), we get $\rho_{\mathrm{Q}}$ in terms of $H$ and

$$
\rho_{\mathrm{Q}}=\frac{1}{8 \pi G}\left\{\left(3-\frac{1}{p}\right) H^{2}-\dot{H}\right\} .
$$

Furthermore, the pressure in the present context is given by

$$
p_{\mathrm{Q}}=-\frac{\dot{H}}{8 \pi G}-V(\phi)=-\frac{1}{8 \pi G}\left\{\left(3-\frac{1}{p}\right) H^{2}+\dot{H}\right\},
$$

which, along with Equation (62) immediately leads to the corresponding EoS parameter:

$$
\omega_{\mathrm{Q}}=-\frac{\left\{\left(3-\frac{1}{p}\right) H^{2}+\dot{H}\right\}}{\left\{\left(3-\frac{1}{p}\right) H^{2}-\dot{H}\right\}} .
$$

Having set the stage, now we are in a position to show the equivalence between QDE and the generalized holographic dark energy model. The comparison of Equations (62) and (32) immediately leads to the equivalent holographic cut-off $\left(L_{\mathrm{Q}}\right)$ corresponds to the QDE as follows:

$$
\begin{aligned}
\frac{3 c^{2}}{\kappa^{2} L_{\mathrm{Q}}^{2}} & =\frac{1}{8 \pi G}\left\{\left(3-\frac{1}{p}\right)\left(\frac{\dot{L}_{\mathrm{p}}}{L_{\mathrm{p}}}-\frac{1}{L_{\mathrm{p}}}\right)^{2}-\left(\frac{\ddot{L}_{\mathrm{p}}}{L_{\mathrm{p}}}-\frac{\dot{L}_{\mathrm{p}}^{2}}{L_{\mathrm{p}}^{2}}+\frac{\dot{L}_{\mathrm{p}}}{L_{\mathrm{p}}^{2}}\right)\right\} \\
& =\frac{1}{8 \pi G}\left\{\left(3-\frac{1}{p}\right)\left(\frac{\dot{L}_{\mathrm{f}}}{L_{\mathrm{f}}}+\frac{1}{L_{\mathrm{f}}}\right)^{2}-\left(\frac{\ddot{L}_{\mathrm{f}}}{L_{\mathrm{f}}}-\frac{\dot{L}_{\mathrm{f}}^{2}}{L_{\mathrm{f}}^{2}}-\frac{\dot{L}_{\mathrm{f}}}{L_{\mathrm{f}}^{2}}\right)\right\} .
\end{aligned}
$$

Thereby the QDE can be equivalently mapped to the generalized holographic dark energy model where the cut-off is a function of $L_{\mathrm{p}}, \dot{L}_{\mathrm{p}}$, and $\ddot{L}_{\mathrm{p}}$, or a function of $L_{\mathrm{f}}, \dot{L}_{\mathrm{f}}$, and $\ddot{L}_{\mathrm{f}}$. Furthermore, the EoS parameter $\left(\Omega_{\mathrm{hol}}^{(Q)}\right)$ corresponding to the hologrphic cut-off $L_{\mathrm{Q}}$ is given by

$$
\Omega_{\mathrm{hol}}^{(Q)}=-1-\left(\frac{2}{3 H L_{\mathrm{Q}}}\right) \frac{d L_{\mathrm{Q}}}{d t}
$$

where $L_{Q}$ is shown above. Clearly, in accordance of Equation (34), $\Omega_{\text {hol }}^{(Q)}$ becomes equivalent to the $\omega_{\mathrm{Q}}$ of Equation (64). Said equivalence leads to the fact that similar to the quintessence energy density, the holographic energy density coming from the cut-off $L_{\mathrm{Q}}$ also provides a good dark energy model of our Universe.

\subsection{Ricci Dark Energy}

In this section, we attempt to establish that the Ricci dark energy (RDE) model has direct equivalence to the generalized holographic dark energy model. The RDE model $[23,29,76]$ catches special attention, as the dark energy density in this context has a geometric origin; in particular, the dark energy density is given by 


$$
\rho_{\mathrm{RD}}=\frac{\alpha}{16 \pi} R=\frac{3 \alpha}{8 \pi}\left(\dot{H}+2 H^{2}\right),
$$

with $R$ being the space-time Ricci scalar and $\alpha$ a model parameter. The above expression of $\rho_{\mathrm{RD}}$ along with its conservation equation lead to the corresponding EoS parameter as

$$
\omega_{\mathrm{RD}}=-1+\frac{\alpha(1+z)}{8 \pi} \frac{d}{d z}\left[\ln \left(\dot{H}+2 H^{2}\right)\right],
$$

where we use the explicit form of $\rho_{\mathrm{RD}}$, and $z=a^{-1}-1$ is known as the red-shift factor. It is evident that the parameter $\alpha$ actually controls the evolution of the $\omega_{\mathrm{RD}}$, and hence the Universe's evolution. In particular, it has been shown in [29] that for $1 / 2<\alpha<1$, the RDE has EoS $-1<\omega_{\mathrm{RD}}<-1 / 3$, and for the case $\alpha<1 / 2$, the RDE starts from quintessence-like and evolves to phantom-like. In regard to the observational compatibility of RDE, the parameter $\alpha$ is constrained by $\alpha=0.394_{-0.106}^{+0.152}$ from SNIa only $(1 \sigma)$; however, a joint analysis of the SNIa+CMB+BAO observations gives a much tighter constraint on $\alpha$ as $\alpha=0.359_{-0.025}^{+0.024}$ [29].

Equations (67) and (32) indicate that the RDE has a direct equivalence to the generalized holographic dark energy model, where the corresponding the cut-off $\left(L_{\mathrm{RD}}\right)$ can be expressed as

$$
\frac{3 c^{2}}{\kappa^{2} L_{\mathrm{RD}}^{2}}=\frac{3 \alpha}{8 \pi}\left\{\frac{\ddot{L}_{\mathrm{p}}}{L_{\mathrm{p}}}+\frac{\dot{L}_{\mathrm{p}}^{2}}{L_{\mathrm{p}}^{2}}-3 \frac{\dot{L}_{\mathrm{p}}}{L_{\mathrm{p}}^{2}}+\frac{2}{L_{\mathrm{p}}^{2}}\right\}
$$

in terms of $L_{\mathrm{p}}, \dot{L}_{\mathrm{p}}$ and $\ddot{L}_{\mathrm{p}}$. Similarly, the $L_{\mathrm{RD}}$ in terms of future horizon and its derivatives is given by

$$
\frac{3 c^{2}}{\kappa^{2} L_{\mathrm{RD}}^{2}}=\frac{3 \alpha}{8 \pi}\left\{\frac{\ddot{L}_{\mathrm{f}}}{L_{\mathrm{f}}}+\frac{\dot{L}_{\mathrm{f}}^{2}}{L_{\mathrm{f}}^{2}}+3 \frac{\dot{L}_{\mathrm{f}}}{L_{\mathrm{f}}^{2}}+\frac{2}{L_{\mathrm{f}}^{2}}\right\} .
$$

Such holographic cut-offs establish symmetry between the RDE and generalized HDE. Furthermore a modified form of RDE has been proposed in [77], where the dark energy density comes with the following form.

$$
\rho_{\mathrm{RD}}=3\left(\alpha H^{2}+\beta \dot{H}\right),
$$

with $\alpha$ and $\beta$ being two parameters. The comparison of the above equation with Equation (32) immediately leads to the equivalence holographic cut-off (in terms of $L_{\mathrm{p}}$ and its derivatives or in terms of $L_{\mathrm{f}}$ and its derivatives) corresponds to the modified RDE $\mathrm{s}$

$$
\begin{aligned}
\frac{3 c^{2}}{\kappa^{2} L_{\mathrm{RD}}^{2}} & =3\left\{\alpha\left(\frac{\dot{L}_{\mathrm{p}}}{L_{\mathrm{p}}}-\frac{1}{L_{\mathrm{p}}}\right)^{2}+\beta\left(\frac{\ddot{L}_{\mathrm{p}}}{L_{\mathrm{p}}}-\frac{\dot{L}_{\mathrm{p}}^{2}}{L_{\mathrm{p}}^{2}}+\frac{\dot{L}_{\mathrm{p}}}{L_{\mathrm{p}}^{2}}\right)\right\} \\
& =3\left\{\alpha\left(\frac{\dot{L}_{\mathrm{f}}}{L_{\mathrm{f}}}+\frac{1}{L_{\mathrm{f}}}\right)^{2}+\beta\left(\frac{\ddot{L}_{\mathrm{f}}}{L_{\mathrm{f}}}-\frac{\dot{L}_{\mathrm{f}}^{2}}{L_{\mathrm{f}}^{2}}-\frac{\dot{L}_{\mathrm{f}}}{L_{\mathrm{f}}^{2}}\right)\right\},
\end{aligned}
$$

where we use Equation (34). The EoS parameter $\left(\Omega_{\mathrm{hol}}^{(R D)}\right)$ corresponds to the holographic energy density $\rho_{\text {hol }}=3 c^{2} /\left(\kappa^{2} L_{\mathrm{RD}}^{2}\right)$ arising from its conservation relation; in particular, we get

$$
\Omega_{\mathrm{hol}}^{(R D)}=-1-\left(\frac{2}{3 H L_{\mathrm{RD}}}\right) \frac{d L_{\mathrm{RD}}}{d t},
$$

which, due to Equation (34), is clearly equivalent to the $\omega_{\mathrm{RD}}$ of Equation (68). Thus, similarly to the RDE, the holographic energy density having the cut-off $L_{\mathrm{RD}}$ proves to be a 
viable dark energy model of the Universe in regard to the SNIa+CMB+BAO observations. Therefore, the RDE and the modified RDE may be regarded as certain candidates for the generalized holographic dark energy family, with the respective holographic cut-offs being given by Equations (69), (70), and (72), respectively.

Before concluding, we consider the scale invariant cosmological field equations [88] and investigate their holographic counterparts. Said field equations are given by [88]

$$
\begin{aligned}
H^{2} & =\frac{8 \pi G}{3} \rho-2 H \frac{\dot{\lambda}}{\lambda}, \\
2 \dot{H}+3 H^{2} & =-8 \pi G p-4 H \frac{\dot{\lambda}}{\lambda},
\end{aligned}
$$

where $\rho$ and $p$ represent the energy density and pressure of the matter components. Moreover, $\lambda$ parametrizes the scale invariance, which varies with the expansion of the Universe, i.e., $\lambda=\lambda(a(t))$. Here it may be mentioned that the authors of [88] proposed an inflationary scenario in the context of scale invariance cosmology, in which case the matter components are provided by a slow rolling scalar field, in particular, $\rho=\frac{1}{2} C\left(\dot{\Psi}^{2}+U(\Psi)\right)$ and $p=\frac{1}{2} C\left(\dot{\Psi}^{2}-U(\Psi)\right)$, where $\Psi$ is a scalar field, $U(\Psi)$ is its potential, and $C$ is a constant (for more information about $C$, see [88]). Clearly the field Equations (74) and (75) can be equivalently mapped to the holographic cosmological scenario, where the holographic cut-off and the corresponding EoS parameter are given by

$$
\begin{aligned}
\frac{3 c^{2}}{\kappa^{2} L_{\mathrm{SI}}^{2}} & =\rho(a)-\frac{3}{4 \pi G}\left(\frac{\dot{\lambda}(a)}{\lambda(a)}\right)\left(\frac{\dot{L}_{\mathrm{p}}}{L_{\mathrm{p}}}-\frac{1}{L_{\mathrm{p}}}\right), \\
\text { or }, \frac{3 c^{2}}{\kappa^{2} L_{\mathrm{SI}}^{2}} & =\rho(a)-\frac{3}{4 \pi G}\left(\frac{\dot{\lambda}(a)}{\lambda(a)}\right)\left(\frac{\dot{L}_{\mathrm{f}}}{L_{\mathrm{f}}}+\frac{1}{L_{\mathrm{f}}}\right),
\end{aligned}
$$

and

$$
\Omega_{\mathrm{hol}}^{(S I)}=-1-\left(\frac{2}{3 H L_{\mathrm{SI}}}\right) \frac{d L_{\mathrm{SI}}}{d t} .
$$

Equations (76) and (77) represent the $L_{\mathrm{SI}}$ in terms of $L_{\mathrm{p}}$ (and its derivative) and $L_{\mathrm{f}}$ (and its derivative) respectively. The EoS parameter in Equation (78) satisfies the conservation relation of the holographic energy density $\rho_{\text {hol }}=3 c^{2} /\left(\kappa^{2} L_{\mathrm{SI}}^{2}\right)$. As a whole, the scale invariant cosmological model (described by Equations (74) and (75)) has a holographic counterpart, with the cut-off being given in Equation (78).

At this stage it deserves mentioning that as far as we could see in many examples of modified gravity, scalar-tensor theory, and gravity in relation to fluids, the corresponding FLRW equations can be always mapped to holographic cosmology with specific IR cut-offs. However, the physical nature of such cut-offs remains obscure.

\section{Conclusions}

Dark energy (DE) is one of the most puzzling issues in modern cosmology. In particular, DE may even be an issue of quantum gravity. In this regard, the holographic principle, one of the cornerstones of quantum gravity, plays an important role in describing the dark energy of our Universe. Based on the holographic principle and on the dimensional analysis, the theory of holographic dark energy (HDE) has been formulated, where the dark energy density is proportional to the inverse squared if the infrared cut-off is true. The holographic cut-off is usually considered to be same as the particle horizon or the future horizon. It may be stressed that instead of adding a term into the Lagrangian, the HDE is based on the holographic principle and on the dimensional analysis and this makes the HDE significantly different than the other theory of DE. In [9], a generalized HDE was proposed where the cut-off $\left(L_{\mathrm{IR}}\right)$ was generalized to be a function of the particle horizon $\left(L_{\mathrm{p}}\right)$ and its derivatives of any order, or a function of the future horizon $\left(L_{\mathrm{f}}\right)$ and 
its derivatives of any order: $L_{\mathrm{IR}}=L_{\mathrm{IR}}\left(L_{\mathrm{p}}, \dot{L}_{\mathrm{p}}, \ddot{L}_{\mathrm{p}}, \cdots, L_{\mathrm{f}}, \dot{L}_{\mathrm{f}}, \cdots, a\right)$. Evidently, with such a generalized form of the $L_{\mathrm{IR}}$, the phenomenology of the generalized HDE becomes richer.

Based on the formalism of the generalized HDE, we showed that a wide class of dark energy models can be regarded as different candidates for the generalized holographic dark energy family with respective cut-offs. In this regard, we first considered several entropic DE models, in particular, Tsallis entropic DE, Rényi entropic DE, and SharmaMittal entropic DE, and showed that they are indeed equivalent to the generalized HDE model. The corresponding cut-offs were determined in terms of $L_{\mathrm{p}}$ and $\dot{L}_{\mathrm{p}}$, or in terms of $L_{\mathrm{f}}$ and $\dot{L}_{\mathrm{f}}$, respectively. Such equivalences between the entropic $\mathrm{DE}$ and the generalized HDE were established for two cases: (1) in the first case, the exponents of the respective entropy functions were regarded to be constant, while in the second case (2) the exponents varied with cosmic time; the exponents were considered to depend on the evolving Hubble parameter. Here it may be mentioned that for the entropic DE models, the equivalent holographic cut-offs depend on the first derivative of $L_{\mathrm{p}}$ or $L_{\mathrm{f}}$. Besides such entropic DE models, some other DE models-such as (1) the quintessence model where a minimally coupled scalar field with an exponential potential serves the dark energy density, and (2) the Ricci DE where the space-time curvature provides the dark energy density-have also been proven to be equivalent to the generalized HDE. The equivalent holographic cut-off corresponds to the quintessence. The Ricci DE model depends on either $L_{p}, \dot{L}_{p}$, and $\ddot{L}_{p}$; or $L_{\mathrm{f}}, \dot{L}_{\mathrm{f}}$, and $\ddot{L}_{\mathrm{f}}$. It may be noted that for both the quintessence and Ricci DE models, the equivalent cut-offs depend on the second derivative of $L_{\mathrm{p}}$ or $L_{\mathrm{f}}$, unlike those of the entropic DE models, where as mentioned earlier, the corresponding $L_{\mathrm{IR}}$ depends at most on the first derivative of the $L_{\mathrm{p}}$ or $L_{\mathrm{f}}$, respectively. Finally, it deserves mentioning that in all the cases, we determined the effective EoS parameter for the DE models and the corresponding generalized HDE models, where the EoS parameters are represented by $\omega_{\mathrm{i}}$ and $\Omega_{\mathrm{hol}}^{(i)}$, respectively (where $i$ denotes the various cases we considered). As a result, we found that $\omega_{\mathrm{i}} \equiv \Omega_{\mathrm{hol}}^{(i)}$, which further confirms the equivalence between various DE models and the respective generalized HDE models. This indicates symmetry between the generalized HDE and different DE models.

In summary, a wide class of dark energy models, including the entropic DE models, were found to be equivalent with the generalized HDE, with the corresponding cut-offs being determined in terms of the particle horizon and its derivatives or in terms of the future horizon and its derivatives. However, understanding the choice of a fundamental viable cut-off still remains to be a debatable topic. The comparison of such cut-offs for realistic descriptions of the Universe's evolution may help provide better understanding of the holographic principle. Furthermore, it is interesting to note that recently, holographic inflation [38] was proposed with the above generalized holographic cut-off. Our considerations indicate that similar equivalence may be established between different inflationary theories and the holographic inflationary model with a generalized cut-off.

Author Contributions: Conceptualization, S.N., S.D.O. and T.P.; formal analysis, S.N., S.D.O. and T.P.; investigation, S.N., S.D.O. and T.P.; writing — original draft preparation, S.D.O. and T.P.; writingreview and editing, S.N., S.D.O. and T.P.; supervision, S.N. and S.D.O.; project administration, S.N. and S.D.O.; funding acquisition, S.N. and S.D.O. All authors have read and agreed to the published version of the manuscript.

Funding: This work was supported by the JSPS Grant-in-Aid for Scientific Research (C), number $18 \mathrm{~K} 03615$.

Institutional Review Board Statement: Not applicable.

Informed Consent Statement: Not applicable.

Data Availability Statement: This is a theoretical study and no experimental data has been listed.

Acknowledgments: This work was supported by the JSPS Grant-in-Aid for Scientific Research (C), number 18K03615 (S.N.). 
Conflicts of Interest: The authors declare no conflict of interest.

Appendix A. Detailed Derivations of Extended Cases of Entropic Dark Energy Models Appendix A.1. Derivation of Equation (45)

Tsallis entropy with a varying exponent, in particular, $\delta=\delta(x)$ where $x=H_{1}^{2} / H^{2}$, is given by

$$
S_{T}=\frac{A_{0}}{4 G}\left(\frac{A}{A_{0}}\right)^{\delta(x)}
$$

Therefore,

$$
\begin{aligned}
\frac{d S_{T}}{d t} & =\frac{\partial S}{\partial A} \frac{d A}{d t}+\frac{\partial S}{\partial x} \frac{d x}{d t} \\
& =-\frac{1}{4 G}\left(\frac{8 \pi \dot{H}}{H^{3}}\right)\left(\frac{A}{A_{0}}\right)^{\delta(x)-1}\left\{\delta(x)+\frac{H_{1}^{2}}{H^{2}} \ln \left(\frac{H_{1}^{2}}{H^{2}}\right) \delta^{\prime}(x)\right\} \\
& =-\frac{1}{4 G}\left(\frac{8 \pi}{H^{3}}\right)\left(\frac{H_{1}^{2}}{H^{2}}\right)^{\delta(x)-1}\left\{\delta(x)+\frac{H_{1}^{2}}{H^{2}} \ln \left(\frac{H_{1}^{2}}{H^{2}}\right) \delta^{\prime}(x)\right\} \dot{H},
\end{aligned}
$$

where in the second equality of the above equation, we used $A=4 \pi r_{h}^{2}$ and $r_{h}=H^{-1}$. By using the above expression of $\frac{d S}{d t}$ in the first law of thermodynamics in Equation (7), one gets

$$
\left\{\delta(x)+\frac{H_{1}^{2}}{H^{2}} \ln \left(\frac{H_{1}^{2}}{H^{2}}\right) \delta^{\prime}(x)\right\}\left(\frac{H_{1}^{2}}{H^{2}}\right)^{\delta(x)-1} \dot{H}=-4 \pi G(\rho+p),
$$

with $\rho$ and $p$ representing the energy density and pressure of the matter contents, which obey the conservation relation: $\dot{\rho}+3 H(\rho+p)=0$. In accordance with this conservation relation, we can integrate Equation (A3) as

$$
\int d x x^{\delta(x)-1}\left\{\delta(x)+x \delta^{\prime}(x) \ln x\right\} H \frac{d H}{d x}=\frac{4 \pi G}{3} \rho+\frac{\Lambda}{6},
$$

where $\Lambda$ is the constant of integration. The expression $x=H_{1}^{2} / H^{2}$ immediately yields

$$
H \frac{d H}{d x}=-\frac{H_{1}^{2}}{2 x^{2}}
$$

and by plugging that into Equation (A4), we obtain

$$
\begin{aligned}
-H_{1}^{2} \int d x\left\{\delta(x)+x \delta^{\prime}(x) \ln x\right\} x^{\delta(x)-3} & =\frac{8 \pi G}{3} \rho+\frac{\Lambda}{3} \\
\Rightarrow-\left.H_{1}^{2}\left\{x^{\delta(x)-2}+2 \int d x x^{\delta(x)-3}\right\}\right|_{x=H_{1}^{2} / H^{2}} & =\frac{8 \pi G}{3} \rho+\frac{\Lambda}{3},
\end{aligned}
$$

which is written in Equation (45).

Appendix A.2. Derivation of Equation (47)

Rényi entropy with varying $\delta=\delta(x)$ is given by

$$
S_{\mathrm{R}}=\frac{A_{0}}{G \delta(x)} \ln \left(1+\frac{\delta(x)}{4}\left(\frac{A}{A_{0}}\right)\right) \text {. }
$$

Therefore, 


$$
\begin{aligned}
\frac{d S_{\mathrm{R}}}{d t} & =\frac{\partial S_{R}}{\partial A} \frac{d A}{d t}+\frac{\partial S_{R}}{\partial x} \frac{d x}{d t} \\
& =\frac{1}{4 G} \frac{1}{\left(1+\frac{\delta(x)}{4}\left(\frac{A}{A_{0}}\right)\right)} \frac{d A}{d t}+\frac{A_{0}}{G}\left\{-\frac{\delta^{\prime}(x)}{\delta^{2}(x)} \ln \left(1+\frac{\delta(x)}{4}\left(\frac{A}{A_{0}}\right)\right)+\frac{\delta^{\prime}(x)}{4 \delta(x)\left(1+\frac{\delta(x)}{4}\left(\frac{A}{A_{0}}\right)\right)}\left(\frac{A}{A_{0}}\right)\right\} \frac{d x}{d t} \\
& =\frac{8 \pi}{H^{3}}\left[-\frac{1}{4\left(1+\frac{\delta(x)}{4}\left(\frac{A}{A_{0}}\right)\right)}+\delta^{\prime}(x)\left\{\frac{1}{\delta^{2}(x)} \ln \left(1+\frac{\delta(x)}{4}\left(\frac{A}{A_{0}}\right)\right)-\frac{x}{4 \delta(x)\left(1+\frac{\delta(x)}{4}\left(\frac{A}{A_{0}}\right)\right)}\right\}\right]\left(\frac{\dot{H}}{G}\right)
\end{aligned}
$$

By plugging the above expression into Equation (7), one gets the evolution of $\dot{H}$ in the present case as

$$
\left[\frac{1}{\left(1+\frac{x \delta(x)}{4}\right)}-4 \delta^{\prime}(x)\left\{\frac{1}{\delta^{2}(x)} \ln \left(1+\frac{x \delta(x)}{4}\right)-\frac{x}{4 \delta(x)\left(1+\frac{x \delta(x)}{4}\right)}\right\}\right] \dot{H}=-4 \pi G(\rho+p) .
$$

Using the conservation relation $\dot{\rho}+3 H(\rho+p)=0$, we integrate Equation (A8) to yield

$$
\int d x\left[\frac{1}{\left(1+\frac{x \delta(x)}{4}\right)}-4 \delta^{\prime}(x)\left\{\frac{1}{\delta^{2}(x)} \ln \left(1+\frac{x \delta(x)}{4}\right)-\frac{x}{4 \delta(x)\left(1+\frac{x \delta(x)}{4}\right)}\right\}\right] H \frac{d H}{d x}=\frac{4 \pi G}{3} \rho+\frac{\Lambda}{6},
$$

where $\Lambda$ represents the constant of integration. Due to the expression of $H \frac{d H}{d x}$ shown in Equation (A5), the above equation takes the following form:

$$
H^{2}=\frac{8 \pi G}{3} \rho+\frac{\Lambda}{3}+\left.4 H_{1}^{2} \int^{x} d x x^{-2}\left\{\left(\frac{\frac{\delta(x)}{16}}{1+\frac{\delta(x)}{4} x}\right)+\left(\frac{1}{\delta(x)^{2}} \ln \left(1+\frac{\delta(x)}{4} x\right)-\frac{1}{\delta(x)} \frac{\frac{1}{4} x}{1+\frac{\delta(x)}{4} x}\right) \delta^{\prime}(x)\right\}\right|_{x=\frac{H_{1}^{2}}{H^{2}}},
$$

which is written in Equation (47).

\section{Appendix A.3. Derivation of Equation (49)}

Sharma-Mittal entropy with varying exponent, in particular, with $\alpha=\alpha(x)$ and $\delta=$ constant, is given by

$$
S_{\mathrm{SM}}=\frac{A_{0}}{G \alpha(x)}\left\{\left(1+\frac{\delta}{4}\left(\frac{A}{A_{0}}\right)\right)^{\frac{\alpha(x)}{\delta}}-1\right\} .
$$

Thereby,

$$
\begin{aligned}
\frac{d S_{\mathrm{SM}}}{d t} & =\frac{\partial S_{S M}}{\partial A} \frac{d A}{d t}+\frac{\partial S_{S M}}{\partial x} \frac{d x}{d t} \\
& =\frac{1}{4 G}\left(1+\frac{\delta}{4}\left(\frac{A}{A_{0}}\right)\right)^{\frac{\alpha(x)}{\delta}-1} \frac{d A}{d t} \\
& +\frac{A_{0}}{G}\left[-\frac{\alpha^{\prime}(x)}{\alpha^{2}(x)}\left\{\left(1+\frac{\delta}{4}\left(\frac{A}{A_{0}}\right)\right)^{\frac{\alpha(x)}{\delta}}-1\right\}+\frac{\alpha^{\prime}(x)}{\alpha(x) \delta}\left(1+\frac{\delta}{4}\left(\frac{A}{A_{0}}\right)\right)^{\frac{\alpha(x)}{\delta}} \ln \left(1+\frac{\delta}{4}\left(\frac{A}{A_{0}}\right)\right)\right] \frac{d x}{d t} \\
& =\frac{8 \pi}{H^{3}}\left[-\frac{1}{4}\left(1+\frac{\delta}{4}\left(\frac{A}{A_{0}}\right)\right)^{\frac{\alpha(x)}{\delta}-1}\right. \\
& \left.+\frac{\alpha^{\prime}(x)}{\alpha^{2}(x)}\left\{\left(1+\frac{\delta}{4}\left(\frac{A}{A_{0}}\right)\right)^{\frac{\alpha(x)}{\delta}}-1\right\}-\frac{\alpha^{\prime}(x)}{\alpha(x) \delta}\left(1+\frac{\delta}{4}\left(\frac{A}{A_{0}}\right)\right)^{\frac{\alpha(x)}{\delta}} \ln \left(1+\frac{\delta}{4}\left(\frac{A}{A_{0}}\right)\right)\right]\left(\frac{\dot{H}}{G}\right) .
\end{aligned}
$$


The above expression of $\frac{d S_{S M}}{d t}$ along with the first law of thermodynamics in Equation (7) lead to the following equation of $\dot{H}$ :

$$
\left[\left(1+\frac{\delta x}{4}\right)^{\frac{\alpha(x)}{\delta}-1}-\frac{4 \alpha^{\prime}(x)}{\alpha^{2}(x)}\left\{\left(1+\frac{\delta x}{4}\right)^{\frac{\alpha(x)}{\delta}}-1\right\}+\frac{4 \alpha^{\prime}(x)}{\alpha(x) \delta}\left(1+\frac{\delta x}{4}\right)^{\frac{\alpha(x)}{\delta}} \ln \left(1+\frac{\delta x}{4}\right)\right] \dot{H}=-4 \pi G(\rho+p),
$$

and upon integration, we obtain Equation (49). Here it may be mentioned that to derive Equation (49), we use $H \frac{d H}{d x}=-H_{1}^{2} /\left(2 x^{2}\right)$ and the conservation relation of $\rho$.

\section{References}

1. Hooft, G.T. Dimensional reduction in quantum gravity. arXiv 1993, arXiv:gr-qc/9310026.

2. Susskind, L. The world as a hologram. J. Math. Phys. 1995, 36, 6377-6396. [CrossRef]

3. Witten, E. Anti de Sitter space and holography. arXiv 1998, arXiv:hep-th/9802150.

4. Bousso, R. The holographic principle. Rev. Mod. Phys. 2002, 74, 825. [CrossRef]

5. Li, M. A model of holographic dark energy. Phys. Lett. B 2004, 603, 1-5. [CrossRef]

6. Li, M.; Li, X.-D.; Wang, S.; Wang, Y. Dark energy. arXiv 2001, arXiv:1103.5870.

7. Wang, S.; Wang, Y.; Li, M. Holographic dark energy. Phys. Rep. 2017, 696, 1-57. [CrossRef]

8. Pavón, D.; Zimdahl, W. Holographic dark energy and cosmic coincidence. Phys. Lett. B 2005, 628, 206-210. [CrossRef]

9. Nojiri, S.; Odintsov, S.D. Unifying phantom inflation with late-time acceleration: Scalar phantom-non-phantom transition model and generalized holographic dark energy. Gen. Relat. Gravit. 2006, 38, 1285-1304. [CrossRef]

10. Enqvist, K.; Sloth, M.S. Possible connection between the location of the cutoff in the cosmic microwave background spectrum and the equation of state of dark energy. Phys. Rev. Lett. 2004, 93, 221302. [CrossRef]

11. Zhang, X. Statefinder diagnostic for holographic dark energy model. Int. J. Mod. Phys. D 2005, 14, 1597-1606. [CrossRef]

12. Guberina, B.; Horvat, R.; Štefančić, H. Hint for quintessence-like scalars from holographic dark energy. J. Cosmol. Astropart. Phys. 2005, 2005, 001. [CrossRef]

13. Elizalde, E.; Nojiri, S.; Odintsov, S.D.; Wang, P. Dark energy: Vacuum fluctuations, the effective phantom phase, and holography. Phys. Rev. D 2005, 71, 103504. [CrossRef]

14. Ito, M. Holographic-dark-energy model with non-minimal coupling. EPL Europhys. Lett. 2005, 71, 712. [CrossRef]

15. Gong, Y.; Wang, B.; Zhang, Y.Z. Holographic dark energy reexamined. Phys. Rev. D 2005, 72, 043510. [CrossRef]

16. Saridakis, E.N. Restoring holographic dark energy in brane cosmology. Phys. Lett. B 2008, 660, 138-143. [CrossRef]

17. Gong, Y.; Li, T. A modified holographic dark energy model with infrared infinite extra dimension (s). Phys. Lett. B 2010, 683, 241-247. [CrossRef]

18. Bouhmadi-Lopez, M.; Errahmani, A.; Ouali, T. Cosmology of a holographic induced gravity model with curvature effects. Phys. Rev. D 2011, 84, 083508. [CrossRef]

19. Malekjani, M. Generalized holographic dark energy model in the Hubble length. Astrophys. Space Sci. 2013, 347, 405-410. [CrossRef]

20. Khurshudyan, M.; Sadeghi, J.; Myrzakulov, R.; Pasqua, A.; Farahani, H. Interacting quintessence dark energy models in Lyra manifold. Adv. High Energy Phys. 2014, 2014, 878092. [CrossRef]

21. Khurshudyan, M. Viscous holographic dark energy universe with Nojiri-Odintsov cut-off. Astrophys. Space Sci. 2016, 361, 1-11.

22. Landim, R.C.G. Holographic dark energy from minimal supergravity. Int. J. Mod. Phys. D 2016, 25, 1650050. [CrossRef]

23. Gao, C.; Wu, F.; Chen, X.; Shen, Y.-G. Holographic dark energy model from Ricci scalar curvature. Phys. Rev. D 2009, 79, 043511. [CrossRef]

24. Li, M.; Lin, C.; Wang, Y. Some issues concerning holographic dark energy. J. Cosmol. Astropart. Phys. 2008, 2008, 023. [CrossRef]

25. Anagnostopoulos, F.K.; Basilakos, S.; Saridakis, E.N. Observational constraints on Barrow holographic dark energy. arXiv 2020, arXiv :2005.10302.

26. Zhang, X.; Wu, F.Q. Constraints on holographic dark energy from type Ia supernova observations. Phys. Rev. D 2005, 72, 043524. [CrossRef]

27. Li, M.; Li, X.-D.; Wang, S.; Zhang, X. Holographic dark energy models: A comparison from the latest observational data. J. Cosmol. Astropart. Phys. 2009, 2009, 036. [CrossRef]

28. Feng, C.; Wang, B.; Gong, Y.; Su, R.-K. Testing the viability of the interacting holographic dark energy model by using combined observational constraints. J. Cosmol. Astropart. Phys. 2007, 2007, 005. [CrossRef]

29. Zhang, X. Holographic Ricci dark energy: Current observational constraints, quintom feature, and the reconstruction of scalar-field dark energy. Phys. Rev. D 2009, 79, 103509. [CrossRef]

30. Lu, J.; Saridakis, E.N.; Setare, M.R.; Xu, L. Observational constraints on holographic dark energy with varying gravitational constant. J. Cosmol. Astropart. Phys. 2010, 2010, 031. [CrossRef]

31. Micheletti, S.M.R. Observational constraints on holographic tachyonic dark energy in interaction with dark matter. J. Cosmol. Astropart. Phys. 2010, 2010, 009. [CrossRef] 
32. Huang, Q.G.; Gong, Y. Supernova constraints on a holographic dark energy model. J. Cosmol. Astropart. Phys. 2004, $2004,006$. [CrossRef]

33. Mukherjee, P.; Mukherjee, A.; Jassal, H.K.; Dasgupta, A.; Banerjee, N. Holographic dark energy: Constraints on the interaction from diverse observational data sets. Eur. Phys. J. Plus 2019, 134, 147. [CrossRef]

34. Nojiri, S.; Odintsov, S.D. Covariant generalized holographic dark energy and accelerating universe. Eur. Phys. J. C 2017, 77, 1-8. [CrossRef]

35. Sharif, M.; Saba, S. Tsallis holographic dark energy in $\mathrm{f}(\mathrm{G}, \mathrm{T})$ gravity. Symmetry 2019, 11, 92. [CrossRef]

36. Jawad, A.; Bamba, K.; Younas, M.; Qummer, S.; Rani, S. Tsallis, Rényi and Sharma-Mittal holographic dark energy models in loop quantum cosmology. Symmetry 2018, 10, 635. [CrossRef]

37. Horvat, R. Holographic bounds and Higgs inflation. Phys. Lett. B 2011, 699, 174-176. [CrossRef]

38. Nojiri, S.; Odintsov, S.D.; Saridakis, E.N. Holographic inflation. Phys. Lett. B 2019, 797, 134829. [CrossRef]

39. Paul, T. Holographic correspondence of F (R) gravity with/without matter fields. EPL Europhys. Lett. 2019, 127, 20004. [CrossRef]

40. Bargach, A.; Bargach, F.; Errahmani, A.; Ouali, T. Induced gravity effect on inflationary parameters in a holographic cosmology. Int. J. Mod. Phys. D 2020, 29, 2050010. [CrossRef]

41. Elizalde, E.; Timoshkin, A.V. Viscous fluid holographic inflation. Eur. Phys. J. C 2019, 79, 1-4. [CrossRef]

42. Oliveros, A.; Acero, M.A. Inflation driven by a holographic energy density. EPL Europhys. Lett. 2020, 128, 59001. [CrossRef]

43. Nojiri, S.; Odintsov, S.D.; Oikonomou, V.K.; Paul, T. Unifying holographic inflation with holographic dark energy: A covariant approach. Phys. Rev. D 2020, 102, 023540. [CrossRef]

44. Nojiri, S.; Odintsov, S.D.; Saridakis, E.N. Holographic bounce. Nucl. Phys. B 2019, 949, 114790. [CrossRef]

45. Brevik, I.; Timoshkin, A.V. Viscous fluid holographic bounce. Int. J. Geom. Methods M 2020, 17, 2050023. [CrossRef]

46. Corianò, C.; Frampton, P.H. Holographic principle, cosmological constant and cyclic cosmology. Mod. Phys. Lett. A 2020, 35, 1950355. [CrossRef]

47. Elizalde, E.; Odintsov, S.D.; Oikonomou, V.K.; Paul, T. Extended matter bounce scenario in ghost free f (R, G) gravity compatible with GW170817. Nucl. Phys. B 2020, 954, 114984. [CrossRef]

48. Odintsov, S.D.; Oikonomou, V.K.; Paul, T. From a bounce to the dark energy era with F (R) gravity. arXiv 2020, arXiv:2009.09947.

49. Tsallis, C. Possible generalization of Boltzmann-Gibbs statistics. J. Stat. Phys. 1988, 52, 479-487. [CrossRef]

50. Lyra, M.L.; Tsallis, C. Nonextensivity and multifractality in low-dimensional dissipative systems. Phys. Rev. Lett. 1998, 80, 53. [CrossRef]

51. Wilk, G.; Włodarczyk, Z. Interpretation of the nonextensivity parameter q in some applications of Tsallis statistics and Lévy distributions. Phys. Rev. Lett. 2000, 84, 2770. [CrossRef]

52. Tsallis, C.; Cirto, L.J.L. Black hole thermodynamical entropy. Eur. Phys. J. C 2013, 73, 1-7. [CrossRef]

53. Komatsu, N.; Kimura, S. Entropic cosmology for a generalized black-hole entropy. Phys. Rev. D 2013, 88, 083534. [CrossRef]

54. Barboza, E.M., Jr.; Nunes, R.C.; Abreu, E.M.C.; Neto, J.A. Dark energy models through nonextensive Tsallis' statistics. Phys. A 2015, 436, 301-310. [CrossRef]

55. Lymperis, A.; Saridakis, E.N. Modified cosmology through nonextensive horizon thermodynamics. Eur. Phys. J. C 2018, 78, 1-11. [CrossRef] [PubMed]

56. Saridakis, E.N.; Bamba, K.; Myrzakulov, R.; Anagnostopoulos, F.K. Holographic dark energy through Tsallis entropy. arXiv 2018, arXiv:1806.01301.

57. Sheykhi, A. Modified Friedmann equations from Tsallis entropy. Phys. Lett. B 2018, 785, 118-126. [CrossRef]

58. Artymowski, M.; Mielczarek, J. Quantum Hubble horizon. Eur. Phys. J. C 2019, 79, 1-10. [CrossRef]

59. Abreu, E.M.C.; Neto, J.A.; Mendes, A.C.R.; Bonilla, A. Tsallis and Kaniadakis statistics from a point of view of the holographic equipartition law. EPL 2018, 121, 45002. [CrossRef]

60. Jawad, A.; Iqbal, A. Modified cosmology through Renyi and logarithmic entropies. Int. J. Geom. Methods M 2018, 15, 1850130. [CrossRef]

61. Zadeh, M.A.; Sheykhi, A.; Moradpour, H. Tsallis agegraphic dark energy model. Mod. Phys. Lett. A 2019, 34, 1950086. [CrossRef]

62. da Silva, W.J.C.; Silva, R. Extended ACDM model and viscous dark energy: A Bayesian analysis. arXiv 2019, arXiv:1810.03759.

63. Biro, T.S.; Czinner, V.G. A q-parameter bound for particle spectra based on black hole thermodynamics with Rényi entropy. Phys. Lett. B 2013, 726, 861-865. [CrossRef]

64. Czinner, V.G.; Iguchi, H. Rényi entropy and the thermodynamic stability of black holes. Phys. Lett. B 2016, 752, 306-310. [CrossRef]

65. Komatsu, N. Cosmological model from the holographic equipartition law with a modified Rényi entropy. Eur. Phys. J. C 2017, 77, 1-12. [CrossRef]

66. Moradpour, H.; Bonilla, A.; Abreu, E.M.C.; Neto, J.A. Accelerated cosmos in a nonextensive setup. Phys. Rev. D 2017, 96, 123504. [CrossRef]

67. Moradpour, H.; Sheykhi, A.; Corda, C.; Salako, I.G. Implications of the generalized entropy formalisms on the Newtonian gravity and dynamics. Phys. Lett. B 2018, 783, 82-85. [CrossRef]

68. Moradpour, H.; Moosavi, S.A.; Lobo, I.P.; Morais Graça, J.P.; Jawad, A.; Salako, I.G. Thermodynamic approach to holographic dark energy and the Rényi entropy. Eur. Phys. J. C 2018, 78, 1-6. [CrossRef] 
69. Jahromi, A.S.; Moosavi, S.A.; Moradpour, H.; Morais Graçac, J.P.; Loboc, I.P.; Salakod, I.G.; Jawade, A. Generalized entropy formalism and a new holographic dark energy model. Phys. Lett. B 2018, 780, 21-24. [CrossRef]

70. Masi, M. A step beyond Tsallis and Rényi entropies. Phys. Let. A 2005, 338, 217-224. [CrossRef]

71. Halliwell, J.J. Scalar fields in cosmology with an exponential potential. Phys. Lett. B 1987, 185, 341-344. [CrossRef]

72. Barreiro, T.; Copeland, E.J.; Nunes, N.J. Quintessence arising from exponential potentials. Phys. Rev. D 2000, 61, 127301. [CrossRef]

73. Rubano, C.; Scudellaro, P. On some exponential potentials for a cosmological scalar field as quintessence. Gen. Relativ. Gravit. 2002, 34, 307-328. [CrossRef]

74. Sangwan, A.; Tripathi, A.; Jassal, H.K. Observational constraints on quintessence models of dark energy. arXiv 2018, arXiv:1804.09350.

75. Adak, D.; Ali, A.; Majumdar, D. Late-time acceleration in a slow-moving Galileon field. Phys. Rev. D 2013, 88, 024007. [CrossRef]

76. del Campo, S.; Fabris, J.C.; Herrera, R.; Zimdahl, W. Cosmology with Ricci dark energ. Phys. Rev. D 2013, 87, 123002. [CrossRef]

77. Granda, L.N.; Oliveros, A. Infrared cut-off proposal for the holographic density. Phys. Lett. B 2008, 669, 275-277. [CrossRef]

78. Bekenstein, J.D. Generalized second law of thermodynamics in black-hole physics. Phys. Rev. D 1974, 9, 3292-3300. [CrossRef]

79. Hawking, S.W. Particle creation by black holes. Commun. Math. Phys. 1975, 43, 199-220. [CrossRef]

80. Jacobson, T. Thermodynamics of spacetime: The Einstein equation of state. Phys. Rev. Lett. 1995, 75, 1260. [CrossRef]

81. Padmanabhan, T. Gravity and the thermodynamics of horizons. Phys. Rept. 2005, 406, 49-125. [CrossRef]

82. Padmanabhan, T. Thermodynamical aspects of gravity: New insights. Rep. Prog. Phys. 2010, 73, 046901. [CrossRef]

83. Cai, R.G.; Kim, S.P. First law of thermodynamics and Friedmann equations of Friedmann-Robertson-Walker universe. J. High Energy Phys. 2005, 2005, 050. [CrossRef]

84. Akbar, M.; Cai, R.G. Thermodynamic behavior of the Friedmann equation at the apparent horizon of the FRW universe. Phys. Rev. D 2007, 75, 084003. [CrossRef]

85. Cai, R.G.; Cao, L.M. Unified first law and the thermodynamics of the apparent horizon in the FRW universe. Phys. Rev. D 2007, 75, 064008. [CrossRef]

86. Nojiri, S.; Odintsov, S.D.; Saridakis, E.N.; Myrzakulov, R. Correspondence of cosmology from non-extensive thermodynamics with fluids of generalized equation of state. Nucl. Phys. B 2020, 950, 114850. [CrossRef]

87. Nojiri, S.; Odintsov, S.D.; Saridakis, E.N. Modified cosmology from extended entropy with varying exponent. Eur. Phys. J. C 2019, 79, 1-10. [CrossRef]

88. Maeder, A.; Gueorguiev, V.G. Scale invariance, horizons, and inflation. Mon. Not. R. Astron. Soc. 2021, 504, 4005-4014. [CrossRef] 